\title{
Predictive Factors for Long-Term Postoperative Visual Outcome in Patients with Macula-Off Rhegmatogenous Retinal Detachment Treated with Vitrectomy
}

\author{
Irini Chatziralli ${ }^{\mathrm{a}} \quad$ Alexandros Chatzirallis $^{\mathrm{b}} \quad$ Dimitrios Kazantzis $^{\mathrm{a}} \quad$ Eleni Dimitriou $^{\mathrm{a}}$ \\ Genovefa Machairoudia $^{a}$ George Theodossiadis ${ }^{a}$ Efstratios Parikakis ${ }^{b}$ \\ Panagiotis Theodossiadis ${ }^{a}$ \\ a 2 nd Department of Ophthalmology, University of Athens, Athens, Greece; b 2 nd Department of Ophthalmology, \\ Ophthalmiatrion Athinon, Athens, Greece
}

\section{Keywords}

Rhegmatogenous retinal detachment - Vitrectomy ·

Predictive factors - Optical coherence tomography

\begin{abstract}
Purpose: The purpose of this prospective study was to evaluate potential predictive factors of long-term postoperative outcomes in patients with macula-off rhegmatogenous retinal detachment (RRD) treated with pars plana vitrectomy (PPV). Methods: Participants in this study were 86 patients diagnosed with macula-off RRD, who underwent PPV. Demographic characteristics and preoperative characteristics of RRD were recorded, while best corrected visual acuity (BCVA) was measured preoperatively and at specific postoperative time points ( 6 weeks and 6,12 , and 24 months). In addition, spectral domain-optical coherence tomography (SD-OCT) characteristics at postoperative week 6 were assessed as potential factors affecting the long-term postoperative visual outcome 24 months after PPV for RRD. Results: Increasing age, duration of RD of more than 1 week, presence of proliferative vitreoretinopathy, increasing central retinal thickness, ellipsoid zone disruption, and external limiting membrane disruption were significantly associated with a worse BVCA. BCVA was not associated with gender,
\end{abstract}

lens status, the location of breaks, the gas tamponade agent used in PPV, the presence of subretinal fluid, and intraretinal fluid. Conclusions: It is important to determine predictive factors for visual outcomes in order to inform patients about their prognosis and help in the decision-making process for patient management.

(c) 2021 S. Karger AG, Basel

\section{Introduction}

Rhegmatogenous retinal detachment (RRD) is characterized by separation of the neurosensory retina from the retinal pigment epithelium due to retinal tear and fluid accumulation, which leads to visual loss if left untreated $[1,2]$. The mean annual incidence of RRD in Europe has been estimated to be 13.3 cases per 100,000 inhabitants [3]. Pars plana vitrectomy (PPV) is considered the procedure of choice for the treatment of RRD, especially in pseudophakic eyes or in posterior vitreous detachment (PVD)-related RRD. It is an effective and safe technique with a success rate ranging from 80 to $92 \%$, although the postoperative functional outcome is not always consistent with the anatomical success $[1,2$, $4,5]$. karger@karger.com

(c) 2021 S. Karger AG, Basel

www.karger.com/oph

Karger $\stackrel{2}{*}$
Correspondence to:

Dr. Irini Chatziralli, eirchat@yahoo.gr 
Previous studies have tried to define prognostic factors that may predict the postoperative anatomic and visual outcomes in patients with RRD [6-14]. Specifically, preoperative visual acuity, age, duration of $\mathrm{RD}$, preoperative coexistent choroidal detachment, proliferative vitreoretinopathy (PVR), extent of RD, and ellipsoid zone (EZ) integrity were found to be related to postoperative visual outcome [7]. Additionally, Jaggi et al. [15] found that prolongation of fluorescence lifetimes in the fovea is mainly driven by a loss of macular pigment and it was significantly correlated with visual acuity after successful RRD repair, suggesting that fluorescence lifetime imaging ophthalmoscopy may be useful in the prediction of long-term functional outcomes after macula-off RRD surgery.

Advances in optical coherence tomography (OCT) have led to the introduction of new anatomic measures on spectral-domain OCT (SD-OCT), such as precise evaluation of individual layers and qualitative and quantitative assessment of fluid distribution, as well as inner and outer retinal irregularity, which may predict more accurately treatment success or failure [6-14]. However, most studies have been retrospective and analyzed a limited number of prognostic factors separately.

In light of the above, the purpose of this prospective study was to investigate potential predictive factors of long-term postoperative visual outcomes at 24 months in patients with macula-off RRD treated with PPV.

\section{Methods}

The participants in this prospective study were 86 patients with primary macula-off $\mathrm{RRD}$, who were successfully treated with $\mathrm{PPV}$ by experienced surgeons (P.T., E.P.) at two tertiary ophthalmic centers between June 2016 and March 2018. Initially, 111 patients with macula-off RRD were enrolled into this study, but 9 patients were lost to follow-up, 4 developed ocular hypertension after surgery, and 12 exhibited re-detachment during the follow-up period. Therefore, 86 patients were included in the final analysis, as shown in Figure 1. All included patients had undergone 23G PPV using the Constellation Vision System (Alcon Laboratories, Inc.) without internal limiting membrane peeling and gas tamponade (SF6 or C3F8), with a 24-month follow-up. Patients with simultaneous cataract extraction, retinal abnormalities (such as age-related macular degeneration, diabetic retinopathy, and retinal vein occlusion), ocular inflammation, uncontrolled glaucoma, high myopia $>6 \mathrm{D}$, $\mathrm{PVR}>$ grade $\mathrm{B}$, and repeated $\mathrm{RD}$ operation were excluded from this study.

At baseline (preoperatively), all of the participants underwent best corrected visual acuity (BCVA) measurement by means of Snellen charts, slit-lamp biomicroscopy, and dilated fundoscopy. The demographic characteristics of patients (age and gender), the specific preoperative characteristics of RRD, i.e., the duration of RRD ( $\leq 1$ week or $>1$ week), the presence of PVR, the location of

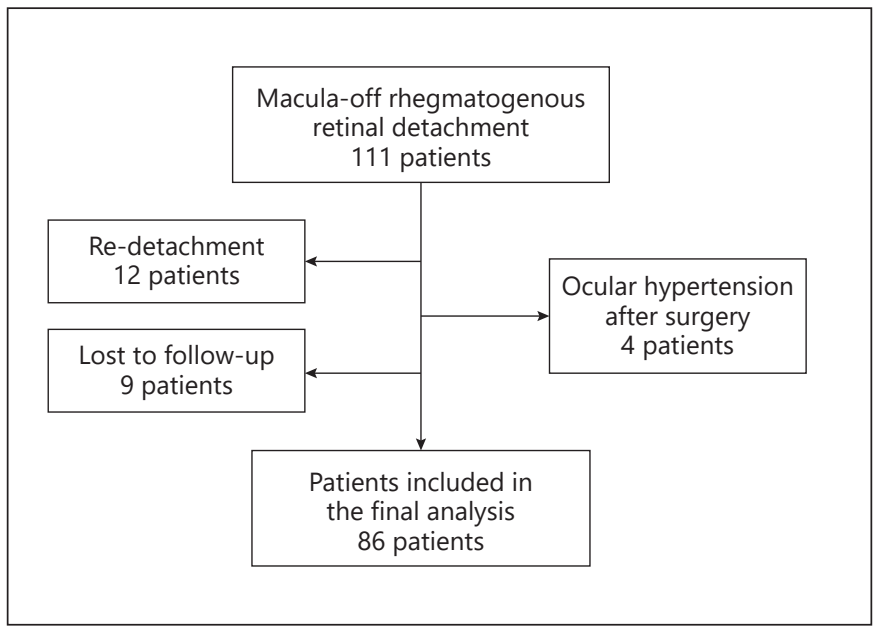

Fig. 1. Flow chart showing the selection of patients in this study.

retinal breaks (superior or inferior), lens status (phakic or pseudophakic), and the gas tamponade agent used for RRD repair (SF6 or C3F8) were recorded. Additionally, BCVA measurement and SDOCT were performed at week 6 postoperatively, so that the gas tamponade agent could be absorbed and visualization of the macula accurately performed, as well as at months 6,12 , and 24 postoperatively. Besides the demographic and baseline clinical characteristics, OCT biomarkers at week 6 were assessed as potential factors affecting the long-term postoperative visual outcome 24 months after PPV for RRD. It is worth noting that OCT was not performed preoperatively since the acquisition could be inaccurate and the measurements not reliable due to the height of the RD.

BCVA was converted to letters for statistical purposes. OCT examination was performed in all cases using SD-OCT (Spectralis HRA + OCT; Heidelberg Engineering, Germany) through dilated pupils. For each patient, 6 radial scans $3 \mathrm{~mm}$ long were performed at equally spaced angular orientations centered on the foveola. OCT scans were evaluated for central retinal thickness (CRT), presence of subretinal fluid (SRF) or intraretinal fluid (IRF), and integrity of the EZ and the external limiting membrane (ELM), defined as intact or disrupted. Two investigators (I.C. and A.C.) independently evaluated the OCT images and the interobserver agreement was almost perfect for all parameters $(k=0.999$ for SRF, $k=0.962$ for IRF, $k=$ 0.913 for EZ condition, and $k=0.891$ for ELM condition).

\section{Statistical Analysis}

For description of the patients' characteristics at baseline, continuous variables were presented as means $( \pm S D)$ and categorical variables were presented as numbers (\%). For longitudinal comparisons of BCVA between baseline and each time point, the Wilcoxon matched-pairs signed-rank test was used; given that $3 \mathrm{com}$ parisons were done, the level of statistical significance was set at $0.05 / 3=0.016$, according to the Bonferroni correction.

For the assessment of factors that may determine visual acuity, since there was a marked deviation from normality, generalized least squares linear regression analysis was performed, as appropriate for longitudinal data, given the intercorrelation of observations in such data sets. Visual acuity was the dependent variable. Factors that were assessed as potential predictors for visual acuity 
were: age, gender, duration of RD, presence of PVR, lens status, gas tamponade agent, CRT, presence of SRF, IRF, and condition of the EZ and ELM. The aforementioned factors were set as independent variables in models always adjusted for time (in months). $\beta$ coefficients with 95\% CI are provided.

Statistical analysis was performed using SPSS 25.0 (IBM Statistics, USA). $p<0.05$ was considered statistically significant, apart from cases where the Bonferroni correction was adopted, as declared above.

\section{Results}

This study included 86 patients ( 86 eyes) with maculaoff primary RDD, with a mean age of $66.2 \pm 8.3$ years. Forty-nine out of 86 patients (57\%) were male and 37 (43\%) were female. Table 1 shows the demographic and clinical baseline characteristics of our study sample.

At baseline, the mean BCVA was $0.97 \pm 0.18 \log$ MAR and it improved to $0.63 \pm 0.11 \log M A R$ at month 6 , to 0.43 $\pm 0.12 \log M A R$ at month 12 , and to $0.40 \pm 0.17 \log$ MAR at month 24 postoperatively, which differed significantly compared to baseline ( $p<0.001$ for all 3 time points).

The results of the generalized least squares linear regression analysis, examining the factors associated with BCVA, are presented in Table 2. Increasing age (coefficient $=-4.11 ; 95 \% \mathrm{CI}=-6.22$ to $-2.47 ; p<0.001)$, duration of RD more than 1 week (coefficient $=+4.14 ; 95 \%$ $\mathrm{CI}=+2.45$ to +5.03 ; $p<0.001$ ), presence of PVR (coefficient $=-3.99 ; 95 \% \mathrm{CI}-6.17$ to $-2.75 ; p<0.001)$, increasing CRT (coefficient $=-2.08 ; 95 \% \mathrm{CI}=-3.59$ to -1.12 ; $p<0.001$ ), EZ disruption (coefficient $=-5.12 ; 95 \% \mathrm{CI}$ -8.13 to $-1.14 ; p<0.001$ ), and ELM disruption (coefficient $=-3.09 ; 95 \% \mathrm{CI}=-5.53$ to $-1.26 ; p<0.001$ ) were significantly associated with a worse BVCA. BCVA was not associated with gender $(p=0.248)$, lens status $(p=$ $0.091)$, location of breaks $(p=0.084)$, gas tamponade agent used in PPV $(p=0.299)$, presence of SRF ( $p=$ $0.064)$, and IRF $(p=0.372)$.

\section{Discussion}

The principal message of this study is that age, duration of RD, presence of PVR, CRT, and condition of the $\mathrm{EZ}$ and the ELM were found to be independent predictive factors of long-term visual outcomes in patients with primary macula-off RRD treated with PPV.

Previous studies have also found that one of the factors determining the postoperative BCVA is the duration of $\mathrm{RD}$, with a longer duration being related to a poorer vi-

Predictive Factors for RRD Treated with PPV
Table 1. Baseline demographic and clinical characteristics of our study sample

\begin{tabular}{lc}
\hline Age, years & $66.2 \pm 8.3$ \\
Gender & \\
$\quad$ Male & $49(57.0)$ \\
$\quad$ Female & $37(43.0)$ \\
BCVA, logMAR & $0.97 \pm 0.18$ \\
Axial length, mm & $24.8 \pm 2.7$ \\
Lens status & \\
$\quad$ Phakic & $52(60.5)$ \\
$\quad$ Pseudophakic & $34(39.5)$ \\
Duration of retinal detachment (weeks) & \\
$\quad \leq 1$ & $69(80.2)$ \\
$\quad>1$ & $17(19.8)$ \\
Proliferative vitreoretinopathy & $13(15.1)$ \\
$\quad$ Yes & $73(84.9)$ \\
$\quad$ No & \\
Location of retinal breaks & $61(70.9)$ \\
$\quad$ Superior & $25(29.1)$ \\
Inferior & $12(14.0)$ \\
Gas tamponade & $74(86.0)$ \\
$\quad$ SF6 &
\end{tabular}

Values are presented as means \pm SD or numbers (\%).

sual acuity $[16,17]$. Of note, Frings et al. [18] suggested that eyes treated within 3 days after the onset of visual loss have a better final postoperative visual acuity than those with a longer lag time. The critical preoperative duration of $\mathrm{RD}$ seems to be 7 days, since several authors have concluded that the duration of macular detachment within the first week does not affect postoperative visual recovery $[7,19,20]$. In our study, RRD with a duration longer than 1 week were found to be associated with poor visual outcomes at 24 months postoperatively.

The integrity of the ELM and that of the EZ are wellknown factors for visual outcomes in several retinal diseases, such as age-related macular degeneration, diabetic macular edema, retinal vein occlusion, and epiretinal membrane [21-26], although it is sometimes difficult to accurately assess the preoperative condition of the ELM and the EZ. Previous studies have found that ELM and EZ integrity has been correlated with postoperative visual outcomes in patients with RD; specifically, disrupted ELM and EZ were associated with poorer postoperative visual outcomes $[7,8,14,27,28]$. Our study has also confirmed the already reported correlation of EZ and ELM with visual acuity.

Our results show that another significant prognostic factor for postoperative visual outcomes in patients with 
Table 2. Results of the generalized least squares linear regression analysis examining the factors associated with visual acuity (in letters)

\begin{tabular}{llrr}
\hline Variable & Category/increment & Coefficient (95\% CI) & $p$ value \\
\hline Age, years & 5-year increase & $-4.11(-6.22$ to -2.47$)$ & $<0.001$ \\
Gender & Male vs. female & $+1.51(-0.63$ to +2.19$)$ & 0.248 \\
Lens status & Phakic vs. pseudophakic & $+2.24(-0.72$ to +4.52$)$ & 0.091 \\
Duration of retinal detachment & $\leq$ 1 vs. $>$ 1 week & $+4.14(+2.45$ to +5.03$)$ & $<0.001$ \\
Presence of proliferative vitreoretinopathy & Yes vs. no & $-3.99(-6.17$ to -2.75$)$ & $<0.001$ \\
Location of retinal breaks & Superior vs. inferior & $+0.32(-0.19$ to +1.02$)$ & 0.084 \\
Gas tamponade & SF6 vs. C3F8 & $+1.47(-0.95$ to +3.13$)$ & 0.299 \\
Central retinal thickness & 50- $\mu$ m increase & $-2.08(-3.59$ to -1.12$)$ & $<0.001$ \\
SRF & Yes vs. no & $+1.08(-0.04$ to +2.13$)$ & 0.064 \\
IRF & Yes vs. no & $-0.67(-1.93$ to +1.39$)$ & 0.372 \\
EZ disruption & Yes vs. no & $-5.12(-8.13$ to -1.14$)$ & $<0.001$ \\
ELM disruption & Yes vs. no & $-3.09(-5.53$ to -1.26$)$ & $<0.001$ \\
\hline
\end{tabular}

All models are adjusted for time (months).

RRD treated with PPV is the presence of PVR, which is in line with previous studies. Pastor et al. [29] found that, in $40-80 \%$ of anatomically successful cases with PVR, patients maintain only ambulatory vision, defined as 5/200 or better.

It is also worth mentioning that the influence of age on postoperative visual acuity in patients with RRD has been previously analyzed in patients treated with scleral buckling, showing that older age is an independent factor of poor postoperative visual outcomes [30], as was found in our study. In addition, it should be noted that older patients are more prone to complications [31].

A quite astonishing finding of our study is that lens status seemed not to impact visual acuity outcomes. This could be attributed to the fact that 30 out of 52 initially phakic patients $(57.7 \%)$ underwent cataract surgery during the follow-up time of 2 years; therefore, since we measured visual acuity at specific time points (months 6,12 , and 24) and cataract surgery was promptly performed when cataract was evident, lens status was found not to affect visual acuity at any time point postoperatively. Similar results have been reported by Halberstadt et al. [32], who also found that the anatomical and functional outcomes of primary RD surgery are similar in phakic and pseudophakic eyes.

A potential limitation of this study pertains to the fact that the accurate duration of RD is based on a subjective symptom (visual acuity or visual field loss) according to patients' reports and not on an objective sign. In addition, no analysis of the speed of recovery of visual acuity was performed. Furthermore, it is worth noting that
BCVA was measured at specific time points (postoperative months 6,12 , and 24), while most of the initially phakic patients underwent cataract surgery between 12 and 24 months of follow-up, thus precluding assessment of the real impact of lens status on postoperative visual acuity. Finally, the nature of the generalized least squares regression models inherently precluded the incorporation of baseline BCVA as a separate covariate in the model.

In conclusion, our study showed that increased age, RRD with a duration of more than one week, coexistent PVR, increased CRT, and disrupted ELM and EZ are independent predictors of poor postoperative visual outcomes in patients with RRD treated with PPV. It is important to determine predictive factors in such patients in order to inform them about their postoperative prognosis and help in the decision-making process in patient management.

\section{Statement of Ethics}

The study was in accordance with the tenets of Helsinki Declaration and it was approved by the institutional review board of the participating hospitals. Inform consent was obtained from all of the participants.

\section{Conflict of Interest Statement}

The authors declare no conflict of interests. 


\section{Funding Sources}

There are no funding sources to declare.

\section{References}

1 Nemet A, Moshiri A, Yiu G, Loewenstein A, Moisseiev E. A Review of Innovations in Rhegmatogenous Retinal Detachment Surgical Techniques. J Ophthalmol. 2017;2017: 4310643.

2 Ah-Fat FG, Sharma MC, Majid MA, McGalliard JN, Wong D. Trends in vitreoretinal surgery at a tertiary referral centre: 1987 to 1996. Br J Ophthalmol. 1999 Apr;83(4):396-8.

3 Li JQ, Welchowski T, Schmid M, Holz FG, Finger RP. Incidence of Rhegmatogenous Retinal Detachment in Europe - A Systematic Review and Meta-Analysis. Ophthalmologica. 2019;242(2):81-6.

4 Fischer N, Loewenstein A. Rhegmatogenous retinal detachment: clinical preoperative and postoperative handling. ESASO Course Series: Surgical Retina. Basel: Karger; 2012. vol 2. p. 99-104.

5 Yoshikawa Y, Shoji T, Kanno J, Ibuki H, Ozaki K, Ishii H, et al. Evaluation of microvascular changes in the macular area of eyes with rhegmatogenous retinal detachment without macular involvement using swept-source optical coherence tomography angiography. Clin Ophthalmol. 2018 Oct;12:2059-67.

6 Lee SH, Han JW, Byeon SH, Kim SS, Koh HJ, Lee SC, et al. Retinal layer segmentation after silicone oil or gas tamponade for macula-on retinal detachment using optical coherence tomography. Retina. 2018 Feb;38(2):310-9.

7 Park DH, Choi KS, Sun HJ, Lee SJ. Factors associated with visual outcome after macula-off rhegmatogenous retinal detachment surgery. Retina. 2018 Jan;38(1):137-47.

8 Kang HM, Lee SC, Lee CS. Association of Spectral-Domain Optical Coherence Tomography Findings with Visual Outcome of Macula-Off Rhegmatogenous Retinal Detachment Surgery. Ophthalmologica. 2015; 234(2):83-90.

9 Kobayashi M, Iwase T, Yamamoto K, Ra E, Murotani K, Matsui S, et al. Association Between Photoreceptor Regeneration and $\mathrm{Vi}$ sual Acuity Following Surgery for Rhegmatogenous Retinal Detachment. Invest Ophthalmol Vis Sci. 2016 Mar;57(3):889-98.

10 Okuda T, Higashide T, Sugiyama K. Metamorphopsia and outer retinal changes after successful vitrectomy surgery for macula-off rhegmatogenous retinal detachment. Retina. 2018 Jan;38(1):148-54.

11 Karacorlu M, Sayman Muslubas I, Hocaoglu M, Arf S, Ersoz MG. Correlation between morphological changes and functional outcomes of recent-onset macula-off rhegmato-

\section{Author Contribution}

I.C. conceived the idea, designed this study, collected data, performed the statistical analysis, interpreted data, and drafted this paper. A.C., D.K., E.D., G.M. and E.P. collected and interpreted data. G.T. interpreted data and critically revised this paper. P.T. collected data and supervised this study. All of the authors read and approved the final version of this work. genous retinal detachment: prognostic factors in rhegmatogenous retinal detachment. Int Ophthalmol. 2018 Jun;38(3):1275-83.

12 Delolme MP, Dugas B, Nicot F, Muselier A, Bron AM, Creuzot-Garcher C. Anatomical and functional macular changes after rhegmatogenous retinal detachment with macula off. Am J Ophthalmol. 2012 Jan;153(1):128-36.

13 Poulsen CD, Petersen MP, Green A, Peto T, Grauslund J. Fundus autofluorescence and spectral domain optical coherence tomography as predictors for long-term functional outcome in rhegmatogenous retinal detachment. Graefes Arch Clin Exp Ophthalmol. 2019 Apr;257(4):715-23.

14 Gharbiya M, Grandinetti F, Scavella V, Cecere M, Esposito M, Segnalini A, et al. Correlation between spectral-domain optical coherence tomography findings and visual outcome after primary rhegmatogenous retinal detachment repair. Retina. 2012 Jan;32(1):43-53.

15 Jaggi D, Solberg Y, Dysli C, Ebneter A, Wolf $\mathrm{S}$, Zinkernagel MS. Fluorescence Lifetime Imaging Ophthalmoscopy: Findings After Surgical Reattachment of Macula-Off Rhegmatogenous Retinal Detachment. Retina. 2020 Oct; 40(10):1929-37.

16 Khanzada MA, Wahab S, Hargun LD. Impact of Duration of Macula off Rhegmatogenous Retinal Detachment on Visual Outcome. Pak J Med Sci. 2014 May;30(3):525-9.

17 Hassan TS, Sarrafizadeh R, Ruby AJ, Garretson BR, Kuczynski B, Williams GA. The effect of duration of macular detachment on results after the scleral buckle repair of primary, macula-off retinal detachments. Ophthalmology. 2002 Jan;109(1):146-52.

18 Frings A, Markau N, Katz T, Stemplewitz B, Skevas C, Druchkiv V, et al. Visual recovery after retinal detachment with macula-off: is surgery within the first $72 \mathrm{~h}$ better than after? Br J Ophthalmol. 2016 Nov;100(11):1466-9.

19 Ross WH. Visual recovery after macula-off retinal detachment. Eye (Lond). 2002 Jul; 16(4):440-6.

20 Burton TC, Lambert RW Jr. A predictive model for visual recovery following retinal detachment surgery. Ophthalmology. 1978 Jun;85(6):619-25.

21 Coscas F, Coscas G, Lupidi M, Dirani A, Srour $\mathrm{M}$, Semoun O, et al. Restoration of Outer Retinal Layers After Aflibercept Therapy in Exudative AMD: prognostic Value. Invest Ophthalmol Vis Sci. 2015 Jun;56(6):4129-34.

22 Zur D, Iglicki M, Busch C, Invernizzi A, Mariussi M, Loewenstein A, et al.; International
Retina Group. OCT Biomarkers as Functional Outcome Predictors in Diabetic Macular Edema Treated with Dexamethasone Implant. Ophthalmology. 2018 Feb;125(2):267-75.

23 Chatziralli I, Theodossiadis G, Chatzirallis A, Parikakis E, Mitropoulos P, Theodossiadis P. RANIBIZUMAB FOR RETINAL VEIN OCCLUSION: Predictive Factors and LongTerm Outcomes in Real-Life Data. Retina. 2018 Mar;38(3):559-68.

24 Chatziralli IP, Sergentanis TN, Sivaprasad S. Hyperreflective foci as independent visual outcome predictor in macular edema due to retinal vascular diseases treated with intravitreal dexamethasone or ranibizumab. Retina. 2016 Dec;36(12):2319-28.

25 Zur D, Iglicki M, Feldinger L, Schwartz S, Goldstein M, Loewenstein A, et al. Disorganization of Retinal Inner Layers as a Biomarker for Idiopathic Epiretinal Membrane After Macular Surgery-The DREAM Study. Am J Ophthalmol. 2018 Dec;196:129-35.

26 Garnavou-Xirou C, Xirou T, Gkizis I, Kabanarou SA, Dimitriou E, Theodossiadis $\mathrm{P}$, et al. The Role of Disorganization of Retinal Inner Layers as Predictive Factor of Postoperative Outcome in Patients with Epiretinal Membrane. Ophthalmic Res. 2020;63(1):13-7.

27 Wakabayashi T, Oshima Y, Fujimoto $\mathrm{H}, \mathrm{Mu}-$ rakami $Y$, Sakaguchi $H$, Kusaka S, et al. Foveal microstructure and visual acuity after retinal detachment repair: imaging analysis by Fourier-domain optical coherence tomography. Ophthalmology. 2009 Mar;116(3):519-28.

28 Benson SE, Schlottmann PG, Bunce C, Xing W, Charteris DG. Optical coherence tomography analysis of the macula after vitrectomy surgery for retinal detachment. Ophthalmology. 2006 Jul;113(7):1179-83.

29 Pastor JC, de la Rúa ER, Martín F. Proliferative vitreoretinopathy: risk factors and pathobiology. Prog Retin Eye Res. 2002 Jan;21(1):127-44.

30 Park SW, Kwon HJ, Byon IS, Lee JE, Oum BS. Impact of Age on Scleral Buckling Surgery for Rhegmatogenous Retinal Detachment. Korean J Ophthalmol. 2017 Aug;31(4):328-35.

31 Anteby R, Barzelay A, Barak A. Vitrectomy in patients 85 years of age and older: surgical outcomes and visual prognosis. Clin Interv Aging. 2018 Feb;13:243-9.

32 Halberstadt M, Chatterjee-Sanz N, Brandenberg L, Koerner-Stiefbold U, Koerner F, Garweg JG. Primary retinal reattachment surgery: anatomical and functional outcome in phakic and pseudophakic eyes. Eye (Lond). 2005 Aug; 19(8):891-8. 